\title{
First successful VLBI observations in the EVN with VIRAC radio telescope $R T-32$
}

\author{
Vladislavs Bezrukovs ${ }^{1}$ \\ Engineering Research Institute, Ventspils International Radioastronomy Centre \\ Inženieru Street 101, Ventspils, LV3600, Latvia \\ E-mail: vladislavsbeventa. IV

\section{Ivar Shmeld} \\ Engineering Research Institute, Ventspils International Radioastronomy Centre \\ Inženieru Street 101, Ventspils, LV3600, Latvia \\ E-mail: ivarsseventa. IV
}

The Irbene radio telescope RT-32 is a fully steerable 32-m parabolic antenna belonging to Ventspils International Radioastronomy Centre (VIRAC). In the past years, the vertex cabin at the secondary focus of the telescope was reconstructed and several receivers were installed. At present, RT-32 allows observations in four different bands: $327 \mathrm{MHz}, 1.6 \mathrm{GHz}, 5 \mathrm{GHz}$ and $12 \mathrm{GHz}$. Two independent VLBI data acquisition systems were installed and tested. The first terminal, TN-16, developed at Niznij Novgorod Radiophysical Research Institute, is dedicated to space debris VLBI radar and ionosphere sounding observations. The second terminal, based on DBBC and Mark5B technology, is a multipurpose data acquisition system compatible with European VLBI network (EVN) requirements. On April 12, 2012, an EVN fringe test experiment was carried out to test the $5 \mathrm{GHz}$ receiver and data acquisition units. Three observatories participated: Onsala, Torun and Irbene. Observations were similar to those in EVN Network Monitoring Experiments with several short 2-4-second scans transmitted to the JIVE correlator during the experiment. Correlation clearly revealed fringes on the two baselines Irbene-Torun and Irbene-Onsala. This paper reviews the current status of the Irbene RT-32 radio telescope, including receivers and data acquisition units available for VLBI observations, and provides details of the fringe test experiment conducted on April 12, 2012.

11th European VLBI Network Symposium \& Users Meeting

October 9-12, 2012

Bordeaux, France

\footnotetext{
${ }^{1}$ Speaker
} 


\section{Introduction}

VIRAC was established in 1994 by a decision of the Latvian Academy of Sciences with the aim to develop research in radio astronomy and astrophysics. The instrumental base for the centre comprised two fully steerable parabolic antennas, RT-16 and RT-32, which have diameters of $16 \mathrm{~m}$ and $32 \mathrm{~m}$, respectively. The telescopes were built in the end of the 1960's and originally used for military purposes. By the time VIRAC was established, both antennas had been in a completely inoperable state. The reconstruction and instrumental refurbishment carried out by the VIRAC engineering team now makes it possible to use radio telescope RT-32 for fundamental and applied scientific research in radio astronomy [1]. The vertex room in the radio telescope secondary focus is fully refurbished, while receiving systems for frequency bands ranging from $327 \mathrm{MHz}$ to $12 \mathrm{GHz}$ have been installed and tuned. A set of recording equipment has already been assembled, which allows recording of the signal in two channels with a bandwidth up to $1 \mathrm{GHz}$. The new recording system provides a high temporal stability, which is a prerequisite for VLBI observations.

At this stage, the scientific objectives of VIRAC are:

- investigation of the motion of objects in the vicinity of the Earth (space debris, satellites, asteroids) and planets;

- study of the Earth's ionosphere and solar radio emission;

- integration into international VLBI networks, including the EVN.

To meet these objectives, the following receiving systems are available:

- $327 \mathrm{MHz}$ (92 cm, P band) - primary focus;

- $\quad 1.6 \mathrm{GHz}(18 \mathrm{~cm}, \mathrm{~L}$ band $)$ - secondary focus;

- $\quad 5 \mathrm{GHz}(6 \mathrm{~cm}, \mathrm{C}$ band $)$ - secondary focus;

- $\quad 6.9-9.3 \mathrm{GHz}(3.2-4.3 \mathrm{~cm})$ - secondary focus;

- $12 \mathrm{GHz}(2.3 \mathrm{~cm})$ - secondary focus.

As noted from this list, all receivers are installed in the secondary focus, except for the $327 \mathrm{MHz}$ one. As a rule, preparation for observations in these bands requires mounting the suitable feed after removal of the previously installed one as well as connection and calibration of the high-frequency unit (RF-unit).

The receiving system for the $12 \mathrm{GHz}$ frequency band is designed to record linearly polarized signals and is used for observations of methanol masers and the Sun.

The spectro-polarimetric receiving complex for the $6.9-9.3 \mathrm{GHz}$ band consists of a broadband feed covering the range $2-18 \mathrm{GHz}$, a multi-spectral polarimeter recording signals in right and left circular polarization, and a data acquisition unit. The system is designed for spectral polarimetric observations of microwave emission of the upper chromosphere, the transient region and the lower corona of the Sun [2].

The receiving units at $327 \mathrm{MHz}, 1.6 \mathrm{GHz}$ and $5 \mathrm{GHz}$ are more versatile and have been designed for VLBI observations. The frequency bands at $327 \mathrm{MHz}$ and $1.6 \mathrm{GHz}$ are employed extensively in VLBI experiments studying the ionosphere and solar activity. The $5 \mathrm{GHz}$ receiving system at present is mainly engaged in locating space debris. It has also been tested in EVN experiments. The aim is to use these receivers in regular EVN sessions in the future. 
For digitalization and recording of the signal, two independent systems are used: TN-16 (developed at the Niznij Novgorod Radiophysical Research Institute), which has a sampling frequency up to $16 \mathrm{MHz}$, and DBBC - the Digital Base Band Converter (developed at the Institute of Radioastronomy in Noto, Italy, for use within the European VLBI community), which is a generic, modular radio astronomical data acquisition system [3].

\section{Receiving system for $5 \mathrm{GHz}$ band}

The receiver for the $5 \mathrm{GHz}$ band used in the EVN fringe test experiment reported below was manufactured in the early 1990's for Medicina Radio Astronomical Observatory. After dismantling, the receiving system was handed over to VIRAC. To operate under proper conditions, the receiver needs cryogenic equipment (currently missing but to be installed in 2013). At present, the receiver is used in a "warm" mode, allowing participation in VLBI experiments, although with reduced sensitivity.

As irradiator, a horn antenna fixed in the secondary focus is used. The feedhorn is connected to the receiver through a waveguide equipped for receiving injected calibration signals. The received signal through the waveguide arrives at the polarizer and enters the orthomode transducer (OMT) which splits radiation into right- and left-polarized components. After amplification and filtering, the signals, with $500 \mathrm{MHz}$ bandwidth in both polarizations, are amplified again and fed to the mixer. When mixed with the local oscillator signal, the input signal is transferred to a lower frequency in the upper side band $(100-450 \mathrm{MHz})$. The amplification of each intermediate frequency signal is performed up to the level of $-20 \mathrm{dBm}$. Example of an intermediate frequency spectrum from one of the channels is shown in Fig. 1. A Rohde \& Schwarz (R\&S) SMP 04 signal generator is used as the local oscillator [4].

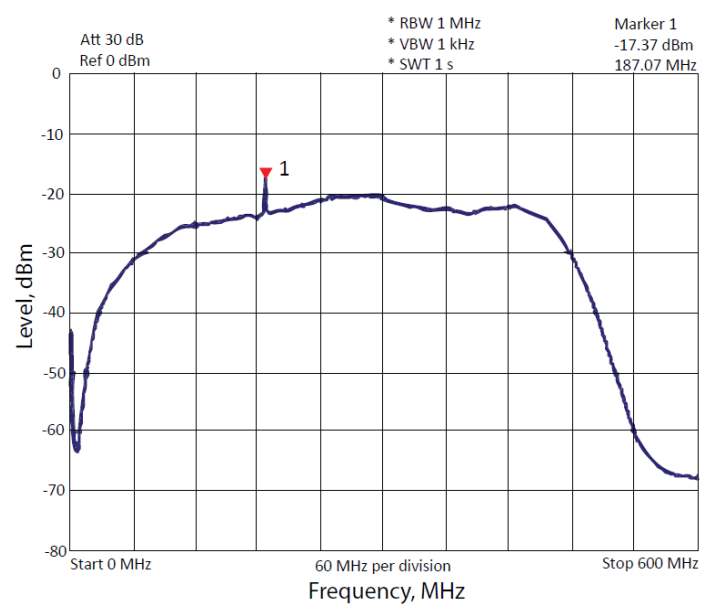

Figure 1. IF spectrum from the LCP channel of the $5 \mathrm{GHz}$ receiver. The IF bandwidth is $100-$ $450 \mathrm{MHz}$ and the local oscillator frequency is set to $4600 \mathrm{MHz}$. The peak corresponds to the test signal at the frequency $4787.07 \mathrm{MHz}$. 


\section{Connection between receiving and recording units}

The connection scheme developed for the EVN fringe test observation described below is shown in Fig. 2. It consists of a $5 \mathrm{GHz}$ receiver unit and a DBBC complemented with Mark5B. For this observation, the second-generation DBBC (DBBC2) was used. DBBC2 can handle up to four IF channels simultaneously; however, it was only possible to record two inputs (IFa and $\mathrm{IFb}$ ) since the Mark5B model installed at the observatory is equipped with a single VSI-H cable.

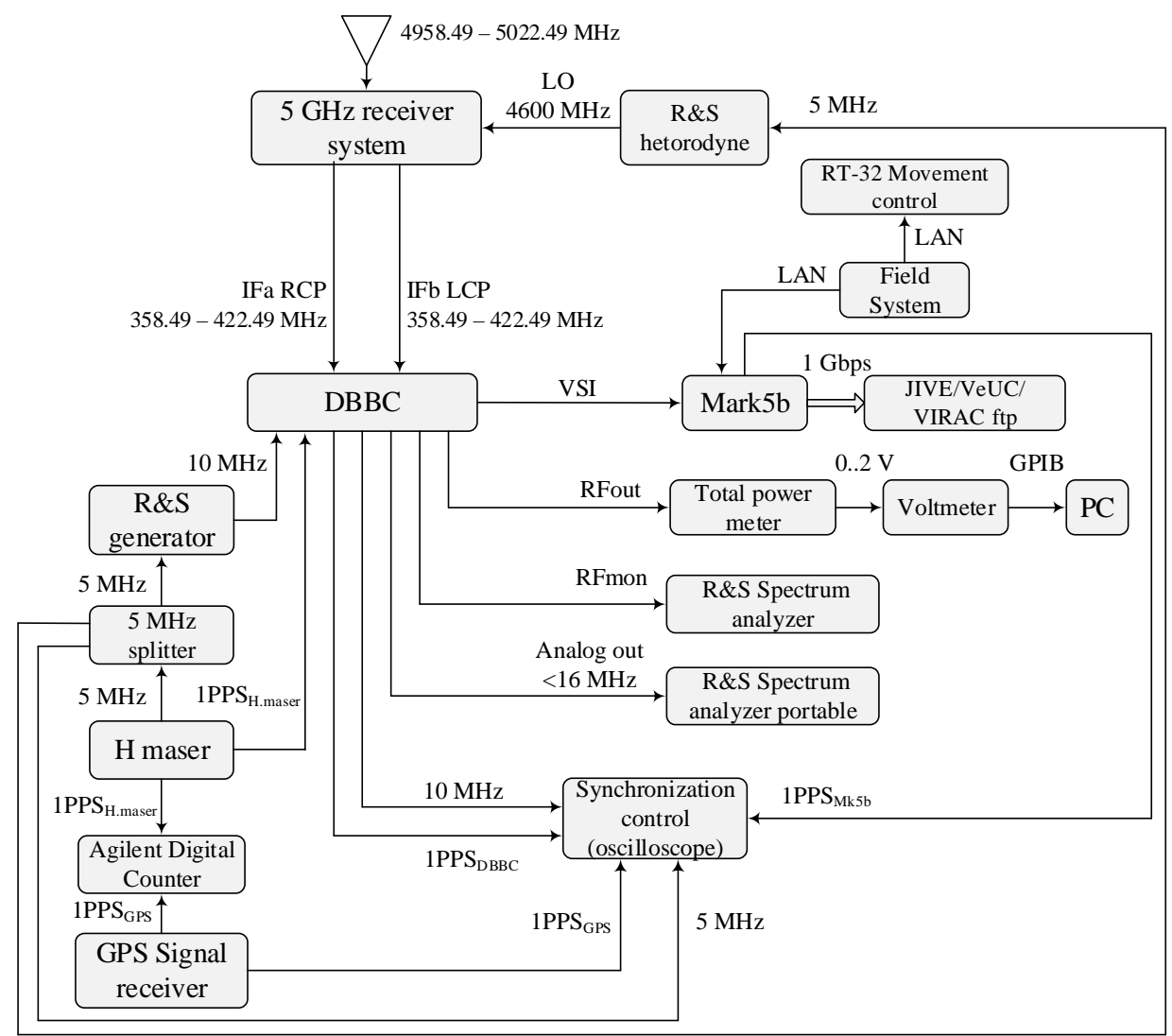

Figure 2. Connection scheme between receiving and recording equipment, as used in the EVN fringe test experiment.

VLBI observations require precise synchronization of the received and sampled data, as well as registration of the exact timestamps. At RT-32, an active hydrogen maser Quartz CH1$75 \mathrm{~A}$ is used as frequency and time standard. This maser generates a reference frequency of $5 \mathrm{MHz}$ and a 1PPS signal, while a Symmetricom XLI Time and Frequency system is employed for setting and checking the time scale. This system generates one-second pulses based on signals from GPS satellites. In addition, it has an NTP-server that provides accurate time transfer to all devices of the telescope via a local network. To control the synchronization of the acquisition system, a four-channel oscilloscope R\&S RTO 1014 is used. This oscilloscope displays the PPS signals from the GPS, DBBC, Mark5B, the $10 \mathrm{MHz}$ signal from the DBBC and the $5 \mathrm{MHz}$ signal from the hydrogen frequency and time standard. The $10 \mathrm{MHz}$ synchronization signal required for the $\mathrm{DBBC}$ is obtained from a signal generator $\mathrm{R} \& \mathrm{~S}$ SMF100A, which in turn is synchronized by the $5 \mathrm{MHz}$ hydrogen maser reference frequency. 
Two spectrum analyzers, R\&S FSP and R\&S FSH8, connected respectively to the DBBC outputs RFmon and AnalogOut, are used in the reception chain of RT-32 for checking the received signal. The use of monitoring equipment permits real-time control of the IF spectrum for the RCP and LCP channels. Additionally, a Mini-Circuit Power detector ZX47-50+, connected to the DBBC RFout, is used to monitor the signal full power. The signal obtained, in the range $0-2 \mathrm{~V}$, is supplied to a digital voltmeter, and then, through the GPIB interface, is fed to a PC fitted with software designed for visualization and recording of the total power.

Control of Mark5B recording and telescope movements is conducted with the "Field System" software. The "Field System" also produces short 2 - 4-second long data files from the recorded scans, which are sent to the JIVE ftp server. This procedure permits correlation of the data in almost real time and achievement of results immediately.

\section{Fringe test experiment: scheduling and results}

As noted above, one of the primary objectives of VIRAC is to join the EVN. To this end, the necessary VLBI equipment was installed over the years. After several attempts, a successful fringe test was achieved during an experiment conducted on April 12, 2012.

The general design of that experiment was that of a normal C-band Network Monitoring Experiment, but with more frequent ftp fringe-test scans. Three radio observatories took part: Onsala, Torun and Irbene. Two different schedules were prepared, each running for the entire 5hour period: one with a single dual-polarization $8 \mathrm{MHz}$ sub-band with $64 \mathrm{Mb} / \mathrm{s}$ data flow (FR012a) and one with eight dual-polarization $8 \mathrm{MHz}$ sub-bands with $512 \mathrm{Mb} / \mathrm{s}$ data flow (FR012b). It was decided to start with the $64 \mathrm{Mb} / \mathrm{s}$ mode because it was simpler, with less chance for mis-mapping of channels and bit-streams, and the lower data rate providing faster ftp transfer and correlation. After several scans were successfully correlated and fringes were achieved, configuration was shifted to the $512 \mathrm{Mb} / \mathrm{s}$ mode in order to test the performance of all BBCs typically used in an astronomy experiment. As observation targets, two strong sources J0237+2848 and 3C84 were used. Each scan transferred to JIVE was 2 to 4 second long.

For the FR012a experiment, the central frequency was set to $4966.49 \mathrm{MHz}$. 22 scans were recorded, 6 of which were ftped. In the FR012b experiment, the central frequencies were set to 4966.49 MHz, 4982.49, 4998.49 and 5014.49 MHz. 20 scans were recorded, 5 of which were ftped. Fringes were obtained in all ftped scans, although not in all channels. For the stronger source, $3 \mathrm{C} 84$, the signal-to-noise ratio was in the range $100-150$ on the baselines Irbene Onsala and Irbene - Torun, while for the fainter source, J0237+2848, it was in the range 12 60. It was found that in Irbene the Mark5B data recording system used in the experiment introduced a 1 -second delay, leading to a loss of $25 \%$ of the data when integrating 4 seconds and a loss of $50 \%$ of the data when integrating 2 seconds. Figure 3 shows two examples of cross-correlation results for the baseline Irbene - Onsala. The left panel plots cross-correlation results for the source 3C84 with 4-second integration time (including $25 \%$ of invalid data for Irbene), while the right panel plots cross-correlation results for the source J0237+2848 with 2second integration time (including 50\% of invalid data for Irbene). The latter has a SNR of 12.44 . 

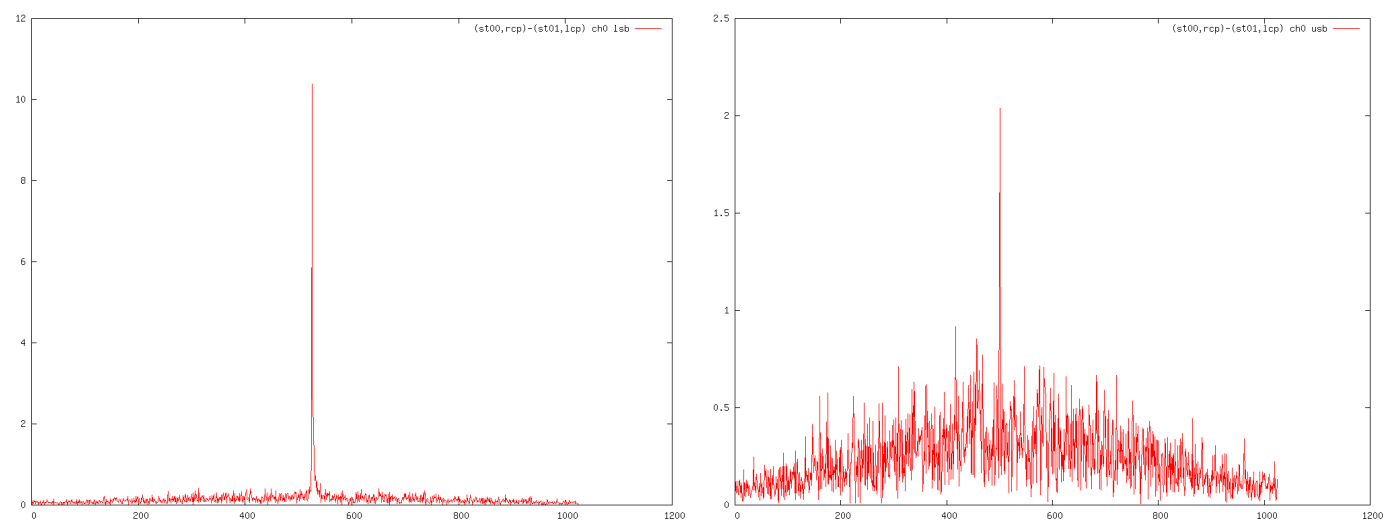

Figure 3. Cross-correlation results for the baseline Irbene (st00, RCP channel) to Onsala (st01, LCP channel). Left panel: source 3C84 with 4-second integration time, SNR 137.3; right panel: J0237+2848 with 2-second integration time, SNR 12.44 .

\section{Conclusions}

The EVN fringe test experiment conducted on April 12, 2012, successfully detected fringes in all scans. However, the experiment also revealed some problems with the Irbene configuration, compared to other station configuration. These include a 1-second delay caused by Mark5B, and LCP and RCP channel swap. These issues were successfully solved afterwards.

Later in the year, Irbene took part in several EVN NME sessions, which identified additional issues that need to be addressed. One of the most important one is the receiver sensitivity, which should be increased by installing a proper cryogenic cooling system. The telescope drive control system also should to be improved. VIRAC engineers are making strong efforts in order to solve these issues and make RT-32 truly available for the EVN community.

\section{Acknowledgements}

The authors wish to express their gratitude for financing to ERDF's project SATTEH, No. 2010/0189/2DP/2.1.1.2.0/10/APIA/VIAA/019, awarded to Engineering Research Institute VIRAC of Ventspils University College. The authors warmly and gratefully remember Guntis Ozolinsh, radio engineer, who made a significant contribution in establishing RT-32, but unfortunately passed away in 2012 .

\section{References}

[1] Bervalds, E., Ozoliņ̌s, G., Sīka, Z., \& Balodis, G., Investigations into the main parameters of the VIRAC 32-metre antenna, Latv. J. Phys. Tech. Sci., 1997, Vol. 2, 48-59.

[2] Bezrukov, D., Spectral polarimetric observations of the Sun by the VIRAC RT-32 radio telescope: first results, Baltic Astronomy, 2013, Vol. 22, 9-14.

[3] Tuccari, G., Alef, W., Bertarini, A., et al., DBBC2 backend: status and development plan, IVS 2010 General Meeting Proceedings, 2010, Eds. D. Behrend and K. D. Baver, NASA/CP2010-215864, 392-395.

[4] Bezrukovs, V., Shmeld, I., Nechaeva, M., et al., Receiving and data acquisition systems of RT-32 for VLBI observations, Latv. J. Phys. Tech. Sci., 2012, Vol. 49, 30-42. 